\title{
Chebyshev Collocation Approach for a Continuous Formulation of Implicit Hybrid Methods for Vips In Second Order Odes
}

\author{
R.B. Adeniyi ${ }^{1}$, E.O. Adeyefa ${ }^{2}$ \\ ${ }^{I}$ Department Of Mathematics, University Of Ilorin, Ilorin, Nigeria. \\ ${ }^{2}$ Department Of Mathematics/Statistics, Federal University Wukari, Taraba State, Nigeria.
}

\begin{abstract}
In this paper, an implicit one-step method for numerical solution of second order Initial Value Problems of Ordinary Differential Equations has been developed by collocation and interpolation technique. The one-step method was developed using Chebyshev polynomial as basis function and, the method was augmented by the introduction of offstep points in order to bring about zero stability and upgrade the order of consistency of the new method. An advantage of the derived continuous scheme is that it can produce several outputs of solution at the off-grid points without requiring additional interpolation. Numerical examples are presented to portray the applicability and the efficiency of the method.
\end{abstract}

Keywords:Interpolation, Chebyshev polynomial, Collocation, continuous scheme.

\section{Introduction} the form:

The general second order Initial Value Problems (IVPs) of Ordinary Differential Equations (ODEs) of

$$
y^{\prime \prime}=f\left(x, y, y^{\prime}\right), y^{\prime}(a)=z_{o}, y(a)=y_{o}, x \in[a, b]
$$

where $f$ is continuous in $[a, b]$, is often encountered in areas such as satellite tracking/warning systems, celestial mechanics, mass action kinetics, solar systems and molecular biology [1]. Many of such problems may not be easily solved analytically, hence numerical schemes are developed to solve (1). These equations are usually reduced to a system of two first order ODEs and numerical methods for first order differential equations are used to solve it. For such systems of first order ODEs, Linear Multistep Methods (LMMs) are powerful numerical methods.

Some researchers have attempted the solution of (1) using LMMs without reduction to system of first order ODEs. They include [2], [3], [4], [5] to mention a few. [6], proposed a continuous scheme based on collocation which was found to have better error estimate and provided approximation at all interior points of the interval of consideration. The main setback of the scheme proposed by [6] is in the need to develop computer sub-programs needed to initialize the starting values; hence, much time is lost and the cost of implementation is high. In view of these disadvantages, many researchers concentrated efforts on advancing the numerical solution of IVPs in ODEs. One of the outcomes is the development of a class of methods called Block method. The method, which shall briefly be discussed in the next section simultaneously generates approximations at different grid points in the interval of integration and is less expensive in terms of the number of function evaluations compared to the LMMs or Runge-Kutta methods.

\section{Block Methods}

Block methods are formulated in terms of LMMs. They provide the traditional advantage of one-step methods, e.g., Runge-Kutta methods, of being self-starting and permitting easy change of step length [7]. Another important feature of the block approach is that all the discrete schemes are of uniform order and are obtained from a single continuous formula in contrast to the non-self starting predictor-corrector approach.

In what now immediately follows, we shall develop the new method with Chebyshev polynomial as basis function.

\section{Development Of The Method}

In this section, we intend to derive a continuous representation of a one-step method which will be used to generate the main method and other methods required to set up the block method. We set out by approximating the analytical solution of problem (1) with a Chebyshev polynomial of the form:

$$
Y(x)=\sum_{j=0}^{k} a_{j} T_{j}(x) \cong y(x)
$$

on the partition

$$
\mathrm{a}=\mathrm{x}_{0}<\mathrm{x}_{\mathrm{l}}<\ldots<\mathrm{x}_{\mathrm{n}}<\mathrm{x}_{\mathrm{n}+1}<\ldots<\mathrm{x}_{\mathrm{N}}=\mathrm{b}
$$

on the integration interval $[a, b]$, with a constant step size $h$, given by $h=x_{n+1}-x_{n} ; n=0,1, \ldots, N-1$. 
Convectionally, we need to interpolate at at least two points to be able to approximate (1) and, to make this happen, we proceed by arbitrarily selecting an offstep point, $x_{n+v}, v \in(0,1)$, in $\left(x_{n}, x_{n+1}\right)$ in such a manner that the zero-stability of the main method is guaranteed. Then (2) is interpolated at $x_{n+i}, i=0, v$ and its second derivative is collocated at $x_{n+1}, i=0, v$ and 1 so as to obtain a system of five equations each of degree four i.e. $k=4$ as follows:

$$
\begin{aligned}
& \sum_{j=0}^{4} a_{j} T_{j}\left(x_{i}\right)=y(x) \\
& \sum_{j=0}^{4} a_{j} T_{j}^{\prime \prime}(x)=f\left(x, y, y^{\prime}\right)
\end{aligned}
$$

In what follows, let us arbitrarily set $v=\frac{1}{2}$. Then, collocating (4) at $x=x_{n+i}, i=0,1$ and $\frac{1}{2}$, and interpolating (3) at $x=x_{n+i}, i=0$ and $\frac{1}{2}$ lead to a system of equations written in the matrix form $\mathrm{AX}=\mathrm{B}$ as:

$$
\left[\begin{array}{ccccc}
1 & -1 & 1 & -1 & 1 \\
1 & 0 & -1 & 0 & 1 \\
0 & 0 & 16 & -96 & 320 \\
0 & 0 & 16 & 0 & -64 \\
0 & 0 & 16 & 96 & 320
\end{array}\right]\left[\begin{array}{c}
a_{0} \\
a_{1} \\
a_{2} \\
a_{3} \\
a_{4}
\end{array}\right]=\left[\begin{array}{c}
y_{n} \\
y_{n+1} \\
h^{2} f_{n} \\
h^{2} f_{n+\frac{1}{2}} \\
h^{2} f_{n+1}
\end{array}\right]
$$

Equation (5) is solved by Gaussian elimination method to obtain the value of the unknown parameters

$$
\begin{aligned}
& a_{0}=y_{n+\frac{1}{2}}+\frac{h^{2}}{256} f_{n}+\frac{7 h^{2}}{128} f_{n+\frac{1}{2}}+\frac{h^{2}}{256} f_{n+1} \\
& a_{1}=y_{n+\frac{1}{2}}-y_{n}+\frac{h^{2}}{64} f_{n}+\frac{5 h^{2}}{48} f_{n+\frac{1}{2}}+\frac{h^{2}}{192} f_{n+1} \\
& a_{2}=\frac{h^{2}}{192} f_{n}+\frac{5 h^{2}}{96} f_{n+\frac{1}{2}}+\frac{h^{2}}{192} f_{n+1} \\
& a_{3}=\frac{h^{2}}{192} f_{n+1}-\frac{h^{2}}{192} f_{n} \\
& a_{4}=\frac{h^{2}}{768} f_{n}-\frac{h^{2}}{384} f_{n+\frac{1}{2}}+\frac{h^{2}}{768} f_{n+1}
\end{aligned}
$$

Substituting (6) into (2) yields a continuous implicit hybrid one-step method in the form:

$$
Y(x)=\alpha_{0}(x) y_{n}+\alpha_{\frac{1}{2}}(x) y_{n+\frac{1}{2}}+h^{2}\left(\sum_{j=0}^{1} \beta_{j}(x) f_{n+j}+\beta_{\frac{1}{2}}(x) f_{n+\frac{1}{2}}\right)
$$

where $\alpha_{j}(x)$ and $\beta_{j}(x)$ are continuous coefficients, $y_{n+j}=y\left(x_{n}+j h\right)$ is the numerical approximation of the analytical solution at $x_{n+j}$ and $f_{n+j}=f\left(x_{n+j}, y_{n+j}, y_{n+j}^{\prime}\right)$.

Equation (7) yields the parameters $\alpha_{j}$ and $\beta_{j}$ as the following continuous function of t:

$$
\left.\begin{array}{l}
\alpha_{0}(t)=-t \\
\alpha_{\frac{1}{2}}(t)=1+t \\
\beta_{0}(t)=\frac{h^{2}}{96}\left(3 t-2 t^{3}+t^{4}\right) \\
\beta_{\frac{1}{2}}(t)=\frac{h^{2}}{48}\left(5 t+6 t^{2}-t^{4}\right) \\
\beta_{1}(t)=\frac{h^{2}}{96}\left(-t+2 t^{3}+t^{4}\right)
\end{array}\right\}
$$

Evaluating (7) at $x_{n+1}$, the main method is obtained as:

$$
y_{n+1}+y_{n}-2 y_{n+\frac{1}{2}}=\frac{h^{2}}{48}\left(f_{n+1}+10 f_{n+\frac{1}{2}}+f_{n}\right)
$$


To derive the block method, additional equations are needed since equation (9) alone will not be sufficient for the solution at $x_{n+\frac{1}{2}}$ and $x_{n+1}$ to be obtained simultaneously. The additional methods can be obtained from evaluating the first derivative of equation (7):

$$
Y^{\prime}(x)=\frac{1}{h}\left[\alpha_{0}^{\prime}(x) y_{n}+\alpha_{\frac{1}{2}}^{\prime}(x) y_{n+\frac{1}{2}}\right]+h\left(\sum_{j=0}^{1} \beta_{j}^{\prime}(x) f_{n+j}+\beta_{\frac{1}{2}}^{\prime}(x) f_{n+\frac{1}{2}}\right)
$$

at $x_{n}, x_{n+\frac{1}{2}}$ and $x_{n+1}$ respectively. This yields the following discrete derivative schemes:

$$
\begin{aligned}
& 48 h y_{n}^{\prime}-96 y_{n+\frac{1}{2}}+96 y_{n}=h^{2}\left(f_{n+1}-6 f_{n+\frac{1}{2}}-7 f_{n}\right) \\
& 48 h y_{n+\frac{1}{2}}^{\prime}-96 y_{n+\frac{1}{2}}+96 y_{n}=h^{2}\left(-f_{n+1}+10 f_{n+\frac{1}{2}}+3 f_{n}\right) \\
& 48 h y_{n+1}^{\prime}-96 y_{n+\frac{1}{2}}+96 y_{n}=h^{2}\left(9 f_{n+1}+26 f_{n+\frac{1}{2}}+f_{n}\right)
\end{aligned}
$$

Equations (9), (11), (12) and (13) are then solved simultaneously to obtain the following explicit results:

$$
\left.\begin{array}{l}
y_{n+\frac{1}{2}}=y_{n}+\frac{1}{2} h y_{n}^{\prime}+\frac{h^{2}}{96}\left(7 f_{n}+6 f_{n+\frac{1}{2}}-f_{n+1}\right) \\
y_{n+1}=y_{n}+h y_{n}^{\prime}+\frac{h^{2}}{6}\left(f_{n}+2 f_{n+\frac{1}{2}}\right) \\
y_{n+\frac{1}{2}}^{\prime}=y_{n}^{\prime}+\frac{h}{24}\left(5 f_{n}+8 f_{n+\frac{1}{2}}-f_{n+1}\right) \\
y_{n+1}^{\prime}=y_{n}^{\prime}+\frac{h}{6}\left(f_{n}+4 f_{n+\frac{1}{2}}+f_{n+1}\right)
\end{array}\right\}
$$

\section{Numerical Examples}

We consider here two test problems for the efficiency and accuracy of the one-step method implemented as a block method.

$$
\begin{aligned}
& \text { 1. } y^{\prime \prime}=y^{\prime}, \quad y(0)=0, y^{\prime}(0)=-1, h=0.1 \\
& \text { Exact Solution }: y(x)=1-\exp (x) \\
& \text { Source }:[5] \\
& y^{\prime \prime}+1001 y^{\prime}+1000 y=0, y(0)=1, y^{\prime}(0)=-1, h=0.05 \\
& \text { Exact Solution }: y(x)=\exp (-x) \\
& \text { Source }:[8]
\end{aligned}
$$

Table 1a: Showing the exact solutions and the computed results from the proposed methods for problem

\begin{tabular}{|l|l|l|}
\hline $\mathrm{X}$ & Exact Solution & The New Method(TNM) \\
\hline 0.1 & -0.105170918 & -0.105170902 \\
\hline 0.2 & -0.221402758 & -0.221402723 \\
\hline 0.3 & -0.349858807 & -0.34985857 \\
\hline 0.4 & -0.491824697 & -0.491824433 \\
\hline 0.5 & -0.64872127 & -0.648720974 \\
\hline 0.6 & -0.8221188 & -0.822118466 \\
\hline 0.7 & -1.013752707 & -1.013752329 \\
\hline 0.8 & -1.225540928 & -1.225540498 \\
\hline 0.9 & -1.459603111 & -1.45960262 \\
\hline 1.0 & -1.718281828 & -1.718281267 \\
\hline
\end{tabular}

Table 1b: Comparing the absolute errors in The New Method (TNM) to error in [5] in problem 1

\begin{tabular}{|l|l|l|}
\hline$X$ & Error in TNM, $\mathrm{p}=4, \mathrm{k}=1$ & Error in [5],p=4, $\mathrm{k}=2$ \\
\hline 0.1 & $0.160756 \mathrm{E}-07$ & $0.87931600 \mathrm{E}-04$ \\
\hline 0.2 & $0.351602 \mathrm{E}-07$ & $0.32671800-03$ \\
\hline 0.3 & $0.237576 \mathrm{E}-06$ & $0.22155640 \mathrm{E}-02$ \\
\hline 0.4 & $0.2646413 \mathrm{E}-06$ & $0.48570930 \mathrm{E}-02$ \\
\hline 0.5 & $0.2967001 \mathrm{E}-06$ & $0.90977340 \mathrm{E}-02$ \\
\hline
\end{tabular}




\begin{tabular}{|l|l|l|}
\hline 0.6 & $0.3343905 \mathrm{E}-06$ & $0.14391394 \mathrm{E}-01$ \\
\hline 0.7 & $0.3784705 \mathrm{E}-06$ & $0.21437918 \mathrm{E}-01$ \\
\hline 0.8 & $0.4304925 \mathrm{E}-06$ & $0.29898724 \mathrm{E}-01$ \\
\hline 0.9 & $0.4911569 \mathrm{E}-06$ & $0.40300719 \mathrm{E}-01$ \\
\hline 1.0 & $0.561459 \mathrm{E}-06$ & $0.52552130 \mathrm{E}-01$ \\
\hline
\end{tabular}

\section{Table of Results}

Table 2a: Showing the exact solutions and the computed results from the proposed methods for problem 2

\begin{tabular}{|l|l|l|}
\hline$X$ & Exact Solution & The New Method \\
\hline 0.1 & $0.90483742 \mathrm{E}+00$ & $0.90483742+00$ \\
\hline 0.2 & $0.81873075 \mathrm{E}+00$ & $0.81873075 \mathrm{E}+00$ \\
\hline 0.3 & $0.74081822 \mathrm{E}+00$ & $0.74081822 \mathrm{E}+00$ \\
\hline 0.4 & $0.67032005 \mathrm{E}+00$ & $0.67032005 \mathrm{E}+00$ \\
\hline 0.5 & $0.60653066 \mathrm{E}+00$ & $0.60653066 \mathrm{E}+00$ \\
\hline 0.6 & $0.54881163 \mathrm{E}+00$ & $0.54881164 \mathrm{E}+00$ \\
\hline 0.7 & $0.49658530 \mathrm{E}+00$ & $0.49658530 \mathrm{E}+00$ \\
\hline 0.8 & $0.44932896 \mathrm{E}+00$ & $0.44932896 \mathrm{E}+00$ \\
\hline 0.9 & $0.40656965 \mathrm{E}+00$ & $0.40656966 \mathrm{E}+00$ \\
\hline 1.0 & $0.36787944 \mathrm{E}+00$ & $0.36787944 \mathrm{E}+00$ \\
\hline
\end{tabular}

Table 2b: Comparing the absolute errors in the New Method to error in [8] in problem 2

\begin{tabular}{|l|l|l|}
\hline $\mathrm{X}$ & Error in TNM, $\mathrm{p}=4, \mathrm{k}=1$ & Error in $[8], \mathrm{p}=6, \mathrm{k}=5$ \\
\hline 0.1 & $0.23596 \mathrm{E}-09$ & $0.698677 \mathrm{E}-11$ \\
\hline 0.2 & $0.47798 \mathrm{E}-09$ & $0.100275 \mathrm{E}-11$ \\
\hline 0.3 & $0.58172 \mathrm{E}-09$ & $0.785878 \mathrm{E}-11$ \\
\hline 0.4 & $0.73564 \mathrm{E}-09$ & $0.104778 \mathrm{E}-10$ \\
\hline 0.5 & $0.81263 \mathrm{E}-09$ & $0.632212 \mathrm{E}-10$ \\
\hline 0.6 & $0.89403 \mathrm{E}-09$ & $0.100508 \mathrm{E}-10$ \\
\hline 0.7 & $0.99141 \mathrm{E}-09$ & $0.936336 \mathrm{E}-11$ \\
\hline 0.8 & $0.101722 \mathrm{E}-08$ & $0.264766 \mathrm{E}-11$ \\
\hline 0.9 & $0.10406 \mathrm{E}-08$ & $0.106793 \mathrm{E}-10$ \\
\hline 1.0 & $0.107144 \mathrm{E}-08$ & $0.232731 \mathrm{E}-10$ \\
\hline
\end{tabular}

\section{Conclusion}

The desirable property of a numerical solution is to behave like the theoretical solution of the problem which can be seen in the result above. It is obvious from TABLE 1 that the new method is more efficient and accurate. However, even though the multiple finite difference method of [8] seemed to have produced a better results at most of the points of evaluation in TABLE $2 b$, it should be noticed that the method had step number $k$ $=5$ against the new method of step number $\mathrm{k}=1$.

Also, the investigation, through the new method reveals the viability of this approach to solve higher order problems. In view of this, we intend to extend the work to step number $\mathrm{k}=2$ and also consider more offstep points.

\section{References}

[1] Aladeselu, V.A., Improved family of block method for special second orderinitial value problems (I.V.Ps). Journal of the Nigerian Association ofMathematical Physics, 11, 2007,153-158.

[2] Lambert, J.D., Numerical Methods for Ordinary Differential Systems(John Wiley, New York, 1991).

[3] Kayode S. J., An Improved Numerov method for Direct Solution of GeneralSecond Order Initial Value Problems of Ordinary Equations, National MathsCentre proceedings 2005.

[4] Adesanya, A.O., Anake T.A. and Oghonyon, G.J., Continuous implicit method for the solution of general second order ordinary differential equations. J. Nig. Assoc. of Math. Phys. 15, 2009, 71-78.

[5] Yahaya, Y. A. and Badmus, A. M., A Class of Collocation Methods for General Second Order Ordinary Differential Equations. African Journal ofMathematics and Computer Science research vol. 2(4), 2009, 069-072.

[6] Awoyemi, D.O., A class of Continuous Methods for general second orderinitial value problems in ordinary differential equation. International Journal of Computational Mathematics, 72, 1999, 29-37.

[7] Lambert, J.D., Computational Methods in Ordinary Differential Equations. John Wiley, New York, 1973.

[8] Jator, S.N., A Sixth Order Linear Multistep Method for the Direct Solutionof $y^{\prime \prime}=f\left(x, y\right.$, $\left.y^{\prime}\right)$. International Journal of Pure and Applied Mathematics,40(4), 2007, 457-472. 